\title{
A Two-Dimensional Gradient-Elasticity Theory for Woven Fabrics
}

\author{
Francesco dell'Isola - David Steigmann
}

\begin{abstract}
A gradient elasticity theory is proposed for the mechanics of woven fabrics. This is motivated by a series of recent experiments in which strongly localized deformation features are observed in the so-called bias test. Such features are reminiscent of solutions to problems posed in the setting of gradient elasticity. In turn, gradient effects in woven fabrics may be motivated by the presence of a local length scale in the pattern of the weave. The presumed influence of this scale on macroscopic constitutive response leads naturally to a special gradient theory.
\end{abstract}

Keywords Gradient elasticity $\cdot$ Mechanics of woven fabrics

\section{Introduction}

In this work we propose an extension of the model advanced in [1] for woven fabrics. In that work the constitutive response to yarn stretch is taken into account but shear resistance between the yarns is suppressed. The present model accounts for that resistance and also for the in-plane flexural resistances of the yarns together with the gradients of the yarn stretches. The model thus furnishes an example of (two-dimensional) gradient elasticity.

The local oscillatory out-of-plane curvature of the yarns of a woven fabric is known as yarn crimp. As the fabric is stretched in plane, the yarns straighten, or decrimp, and the

F. dell'Isola

Dipartimento di Ingegneria Strutturale e Geotecnica, Universita di Roma 'La Sapienza', 00184 Roma, Italy

D. Steigmann $(\bowtie)$

Department of Mechanical Engineering, University of California, Berkeley, CA 94720, USA

e-mail: steigman@me.berkeley.edu

F. dell'Isola · D. Steigmann

International Research Center for Mathematics and Mechanics of Complex Systems,

Università dell'Aquila, Cisterna di Latina, Italy 
lengths of their projections onto the plane increase [2-4]. We refer to these projected curves as fibers. Rather than model crimp explicitly, we proceed as in $[1,3]$ and instead model its effect; namely, the stretches of the projected fibers. Moreover, on the small scale, the width of a yarn and the half wavelength of the weave are roughly equal, at least in tightly woven fabrics; their common value furnishes a local length scale that leads naturally to a gradient effect in the constitutive theory. This manifests itself in certain strongly localized features of the deformation of fabrics observed in the so-called bias test [5]. A relatively simple strainenergy function is proposed to account for these effects. This presumes the local length scale to be small in comparison to the local deformation-induced in-plane radius of curvature of a fiber or the characteristic length for spatial variations of the fiber stretches.

In Sect. 2 we develop the kinematical foundation of the model and interpret it in terms of the differential geometry of the deformed fiber network. Section 3 is concerned with the constitutive structure; in particular, the strain-energy function and associated response functions that are needed in the equations of equilibrium. The latter are derived by a simple variational argument in the Appendix. We also present several remarks intended to motivate our particular choice of constitutive function.

A combination of direct notation and standard Cartesian tensor notation is used. The tensor product of vectors is indicated by interposing the symbol $\otimes$, and the Euclidean inner product of tensors $\mathbf{A}, \mathbf{B}$ is denoted by $\mathbf{A} \cdot \mathbf{B}=\operatorname{tr}\left(\mathbf{A} \mathbf{B}^{t}\right)$; the associated norm is $|\mathbf{A}|=\sqrt{\mathbf{A} \cdot \mathbf{A}}$. The notation $F_{\mathbf{A}}$ stands for the tensor-valued derivative of a scalar-valued function $F(\mathbf{A})$.

\section{Kinematics}

\subsection{Fiber Decompositions of the First and Second Deformation Gradients}

For the sake of simplicity, and with no significant loss of generality, we confine attention to two fiber families that form a uniform orthogonal net in the reference configuration, which is taken to be the initial configuration of the fabric. The frictional interaction between yarns of the weave is presumed to be sufficient to prevent relative slipping of the fibers. In effect, fibers are presumed to be connected to each other at internal pivots which play the role of material points in the continuum theory (see [6], in which this restriction is relaxed).

We have in mind the idea that the constitutive response of the fabric should reflect the underlying responses of the individual fiber families in addition to their near-range interactions induced by their connectivity. The fibers are regarded as elasticae that respond to stretch, bending (curvature induced by deformation) and (tangential) stretch gradient along the fiber. These effects are intrinsic to a given fiber and emerge naturally in a theory of single polymer fibers. The stretching and bending resistance are intuitively clear, whereas sensitivity to the tangential gradient of stretch is associated with finite-width effects [7, 8]. For fabrics, a further constitutive sensitivity to the gradient of stretch orthogonal to the fibers is motivated below.

For example, the stretch and orientation of a particular fiber are given respectively by

$$
\lambda=|\mathbf{d}| \quad \text { and } \quad \boldsymbol{\tau}=\lambda^{-1} \mathbf{d},
$$

where

$$
\mathbf{d}=\mathbf{F D},
$$

in which $\mathbf{D}$ is the unit tangent to the fibre trajectory in the reference configuration, and

$$
\mathbf{F}=\nabla \chi
$$


is the gradient of the deformation function $\chi(\mathbf{X})$. The formula (2) is easily derived by taking the derivative of $\mathbf{r}(S)=\chi(\mathbf{X}(S))$, upon making the identifications $\mathbf{D}=\mathbf{X}^{\prime}(S)$ and $\mathbf{d}=\mathbf{r}^{\prime}(S)$.

Here and henceforth primes refer to derivatives with respect to arclength along a fiber in the reference configuration.

We have $\mathbf{X}=X_{A} \mathbf{E}_{A}$ and $\mathbf{d}=d_{i} \mathbf{e}_{i}$, where

$$
d_{i}=F_{i A} D_{A}
$$

and $\left\{\mathbf{e}_{i}\right\},\left\{\mathbf{E}_{A}\right\}$ are similarly oriented orthonormal bases, assumed here to coincide.

We use the notation $\mathbf{D}_{\alpha}(\alpha=1,2)$ to identify the initial fiber trajectories. Orthogonality of the fiber network prior to deformation implies that the $\mathbf{D}_{\alpha}$ are orthonormal vectors. The presumed uniformity of the network then implies that they form a constant orthonormal basis; we use it to write the two-dimensional identity in the form

$$
\mathbf{I}=\mathbf{D}_{1} \otimes \mathbf{D}_{1}+\mathbf{D}_{2} \otimes \mathbf{D}_{2}, \quad \text { or } \quad \delta_{A B}=D_{A}^{(1)} D_{B}^{(1)}+D_{A}^{(2)} D_{B}^{(2)} .
$$

Extensions of the theory to accommodate non-orthogonal or non-uniform meshes are straightforward, but the present restriction suffices to reveal the important aspects of the theory and to address the more common applications.

Using obvious notation, we combine (2) with $\mathbf{F}=\mathbf{F I}$; i.e.,

$$
F_{i A}=F_{i B} \delta_{B A},
$$

to obtain the fiber decomposition

$$
\mathbf{F}=\mathbf{d}_{1} \otimes \mathbf{D}_{1}+\mathbf{d}_{2} \otimes \mathbf{D}_{2}
$$

of the deformation gradient. Thus,

$$
\mathbf{d}_{1}=\mathbf{F D}_{1} \quad \text { and } \quad \mathbf{d}_{2}=\mathbf{F D}_{2} .
$$

The fiber decomposition of the Cauchy-Green deformation tensor $\mathbf{C}=\mathbf{F}^{t} \mathbf{F}$ is

$$
\mathbf{C}=\lambda_{1}^{2} \mathbf{D}_{1} \otimes \mathbf{D}_{1}+\lambda_{2}^{2} \mathbf{D}_{2} \otimes \mathbf{D}_{2}+S\left(\mathbf{D}_{1} \otimes \mathbf{D}_{2}+\mathbf{D}_{2} \otimes \mathbf{D}_{1}\right),
$$

where

$$
\lambda_{\alpha}=\left|\mathbf{d}_{\alpha}\right| \quad \text { and } \quad S=\mathbf{d}_{1} \cdot \mathbf{d}_{2}=\lambda_{1} \lambda_{2} \sin \gamma
$$

are the fiber extensions and fiber shear strain, respectively, and $\gamma$ is the fiber shear angle, given by [9-12]

$$
\sin \gamma=\boldsymbol{\tau}_{1} \cdot \boldsymbol{\tau}_{2}
$$

Our model presumes the fibers to be constitutively sensitive to bending strain and the gradient of fiber stretch. For a given fiber, the bending strain and the tangential component of the stretch gradient are derived from (cf. (1))

$$
\lambda^{\prime} \boldsymbol{\tau}+\lambda \kappa \boldsymbol{v}=\mathbf{g}, \quad \text { where } \mathbf{g}=\mathbf{r}^{\prime \prime},
$$

in which

$$
\kappa \boldsymbol{v}=\boldsymbol{\tau}^{\prime} \quad \text { and } \quad \boldsymbol{v}=\mathbf{k} \times \boldsymbol{\tau} .
$$


Here $\mathbf{k}$ is the unit normal to the plane of deformation, oriented such that $\mathbf{k} \cdot \mathbf{D}_{1} \times \mathbf{D}_{2}=1$, and $\kappa$ is the bending strain. The in-plane (geodesic) curvature of the fiber after deformation is denoted by $\eta$ and given by $\kappa=\lambda \eta$. The fibre stretch gradient $\lambda^{\prime}$ and bending strain $\kappa$ are therefore obtained from an orthogonal decomposition of $\mathbf{r}^{\prime \prime}$. On the other hand,

$$
\mathbf{g}=\mathbf{F}^{\prime} \mathbf{D}
$$

or, in terms of components,

$$
g_{i}=F_{i A}^{\prime} D_{A}=G_{i A B} D_{A} D_{B}
$$

where

$$
G_{i A B}=F_{i A, B}
$$

is the second gradient of the deformation. The compatibility condition on $\mathbf{F}$ is given simply by

$$
G_{i A B}=G_{i B A} .
$$

Using the identity

$$
G_{i A B}=G_{i C D} \delta_{C A} \delta_{D B}
$$

together with formulas like (15), we obtain the fiber decomposition

$$
G_{i A B}=g_{i}^{(1)} D_{A}^{(1)} D_{B}^{(1)}+g_{i}^{(2)} D_{A}^{(2)} D_{B}^{(2)}+\Gamma_{i}\left(D_{A}^{(1)} D_{B}^{(2)}+D_{A}^{(2)} D_{B}^{(1)}\right),
$$

where

$$
\Gamma_{i}=G_{i C D} D_{C}^{(2)} D_{D}^{(1)}=G_{i C D} D_{C}^{(1)} D_{D}^{(2)},
$$

the final equality being a consequence of (17). In direct notation,

$$
\mathbf{G}=\mathbf{g}_{1} \otimes \mathbf{D}_{1} \otimes \mathbf{D}_{1}+\mathbf{g}_{2} \otimes \mathbf{D}_{2} \otimes \mathbf{D}_{2}+\boldsymbol{\Gamma} \otimes\left(\mathbf{D}_{1} \otimes \mathbf{D}_{2}+\mathbf{D}_{2} \otimes \mathbf{D}_{1}\right),
$$

where

$$
\mathbf{g}_{1}=\left\{\nabla\left(\mathbf{F D}_{1}\right)\right\} \mathbf{D}_{1}, \quad \mathbf{g}_{2}=\left\{\nabla\left(\mathbf{F D}_{2}\right)\right\} \mathbf{D}_{2} \quad \text { and } \quad \boldsymbol{\Gamma}=\left\{\nabla\left(\mathbf{F} \mathbf{D}_{1}\right)\right\} \mathbf{D}_{2}=\left\{\nabla\left(\mathbf{F D}_{2}\right)\right\} \mathbf{D}_{1} .
$$

Thus,

$$
\mathbf{G D}_{1}=\mathbf{g}_{1} \otimes \mathbf{D}_{1}+\boldsymbol{\Gamma} \otimes \mathbf{D}_{2} \quad \text { and } \quad \mathbf{G D}_{2}=\mathbf{g}_{2} \otimes \mathbf{D}_{2}+\boldsymbol{\Gamma} \otimes \mathbf{D}_{1},
$$

which may be compared to (7).

2.2 Differential Geometry of the Deformed Fabric

To aid in the interpretation of the variables used, we introduce fiber angles $\theta_{\alpha}$ such that, for a given fiber,

$$
\boldsymbol{\tau}=\cos \theta \mathbf{D}_{1}+\sin \theta \mathbf{D}_{2}, \quad \boldsymbol{v}=-\sin \theta \mathbf{D}_{1}+\cos \theta \mathbf{D}_{2} .
$$

Then the fiber bending strain is $\kappa=\theta^{\prime}$; using the notation $(\cdot)_{, \alpha}=\partial(\cdot) / \partial \xi_{\alpha}$, where $\xi_{\alpha}$ are Cartesian coordinates aligned with the fiber axes $\left\{\mathbf{D}_{\alpha}\right\}$, we have

$$
\boldsymbol{\tau}_{1,1}=\kappa_{1} \boldsymbol{v}_{1} \quad \text { and } \quad \boldsymbol{\tau}_{2,2}=\kappa_{2} \boldsymbol{v}_{2}, \quad \text { where } \kappa_{1}=\theta_{1,1} \text { and } \kappa_{2}=\theta_{2,2} \text {. }
$$


Further, from (11) it follows that

$$
\theta_{2}-\theta_{1}=\frac{\pi}{2}-\gamma
$$

where $\gamma$ is the fiber shear angle. The determinant of the deformation gradient is

$$
J=\operatorname{det} \mathbf{F}=\lambda_{1} \lambda_{2} \cos \gamma,
$$

provided that $\cos \gamma>0$. This is given in terms of the shear strain $S$ by

$$
J^{2}=\lambda_{1}^{2} \lambda_{2}^{2}-S^{2} .
$$

The compatibility condition (17) is equivalent to the two equalities in $(22)_{3}$; i.e., to

$$
\mathbf{d}_{1,2}=\boldsymbol{\Gamma}=\mathbf{d}_{2,1},
$$

where, from (1) and (24),

$$
\mathbf{d}_{1,2}=\lambda_{1,2} \boldsymbol{\tau}_{1}+\lambda_{1} \theta_{1,2} \boldsymbol{v}_{1} \quad \text { and } \quad \mathbf{d}_{2,1}=\lambda_{2,1} \boldsymbol{\tau}_{2}+\lambda_{2} \theta_{2,1} \boldsymbol{v}_{2} .
$$

The derivatives $\theta_{1,2}$ and $\theta_{2,1}$ are the Tchebychev curvatures of the fibers $[12,13]$. We express them in terms of the bending strains and the derivatives of the fiber shear angle by using $\nabla \gamma=\nabla \theta_{1}-\nabla \theta_{2}$, where $\nabla \theta_{\alpha}=\left(\mathbf{D}_{\beta} \cdot \nabla \theta_{\alpha}\right) \mathbf{D}_{\beta}$ :

$$
\theta_{1,2}=\kappa_{2}+\gamma_{, 2} \quad \text { and } \quad \theta_{2,1}=\kappa_{1}-\gamma_{, 1} .
$$

Projection of the compatibility condition (29) onto $\boldsymbol{\tau}_{1}$ and $\boldsymbol{\tau}_{2}$ furnishes

$$
\lambda_{2} \theta_{2,1} \cos \gamma=\lambda_{2,1} \sin \gamma-\lambda_{1,2} \quad \text { and } \quad \lambda_{1} \theta_{1,2} \cos \gamma=\lambda_{2,1}-\lambda_{1,2} \sin \gamma,
$$

and with (31) these reduce to relations involving the stretches, the bending strains and the shear angle. Because these are satisfied, the term $\boldsymbol{\Gamma}$ in (21) may be identified with either of Eq. (29). Writing them as a system for the derivatives $\lambda_{1,2}$ and $\lambda_{2,1}$, we find that the latter vanish if and only if both Tchebychev curvatures vanish, provided that $J \neq 0$. In this work we enforce the latter requirement by stipulating that the strain-energy function become unbounded as $J \rightarrow 0$ through positive values, thereby effectively penalizing fiber collapse; in this case it follows that $\boldsymbol{\Gamma}$ is determined by $\gamma, \lambda_{1,2}$ and $\lambda_{2,1}$ and that $\boldsymbol{\Gamma}$ vanishes if and only if $\lambda_{1,2}$ and $\lambda_{2,1}$ vanish together.

In a coarse-mesh model of discrete fibers connected at internal pivots so as to form a pantographic lattice, there is no analogue of the transverse fiber derivatives $\lambda_{1,2}$ and $\lambda_{2,1}$ (although $\lambda_{1,1}$ and $\lambda_{2,2}$ remain well defined). In a continuum model of such lattices it would be natural to suppose that the network is constitutively insensitive to $\lambda_{1,2}$ and $\lambda_{2,1}$, and hence insensitive to $\boldsymbol{\Gamma}$. Woven fabrics are quite different due to the fact that the crimp wavelength and the width of an individual yarn are of the same order, whereas in pantographs the spacing between the pivots where the fibers intersect is typically at least an order of magnitude larger than a typical fiber width. Accordingly, to distinguish fabrics from pantographs in the present framework, it would be natural to retain a constitutive sensitivity to $\boldsymbol{\Gamma}$.

In the case of fiber inextensibility, where the stretch gradients vanish identically, $\boldsymbol{\Gamma}$ also vanishes, yielding the further simplifications $\kappa_{1}=\gamma_{, 1}$ and $\kappa_{2}=-\gamma_{, 2}$, connecting the bending strains to the fiber derivatives of the shear angle (see also [14, 15]). Stiff pantographs are well modeled by this idealization $[16,17]$, but woven fabrics are different: The crimp 
mechanism in the kinematics of an actual weave of a woven fabric are represented-in a two-dimensional theory - by the stretches of the fiber projections onto the plane, and so woven fabrics and pantographic lattices are fundamentally distinct insofar as their mechanical behavior is concerned.

\section{Model Strain-Energy Functions}

We propose that the mechanical response of the fabric is adequately described by a strainenergy function $W(\mathbf{F}, \mathbf{G})$, which is presumed to depend on $\mathbf{X}$ only implicitly-via its arguments - to reflect the presumed uniformity of the material properties. Scale effects in the material response arise from the constitutive dependence on $\mathbf{G}$. In the specialization of the model discussed here, we assume $l|\mathbf{G}|$ to be small, where $l$ is a material length scale such as the width of a yarn or the wavelength of the weave. These are essentially equivalent in densely woven fabrics. Our assumption ensures that the radius of curvature of a bent yarn is much larger than its width, and that the characteristic length for spatial variations of the crimp-induced yarn stretches is much larger than the wavelength of the weave. In general, some caution is required when applying this kind of reasoning. For example, the work of dell'Isola and Seppecher $[14,15]$ indicates that the relationship between microstructural dimensions and the stiffness of microstructural elements is crucial in arriving at the constitutive response of the associated homogenized continuum.

If the strain energy and its derivatives vanish in the absence of deformation, then the leading-order strain energy function consistent with our hypotheses is of the form

$$
W(\mathbf{F}, \mathbf{G})=W_{1}(\mathbf{F})+W_{2}(\mathbf{F}, \mathbf{G}),
$$

where $W_{2}$ is homogeneous of degree two in $\mathbf{G}$.

\subsection{Two Strain-Energy Functions}

(a) A strain-energy function based on the fiber decomposition.

The structure of the fiber decompositions of $\mathbf{F}$ and $\mathbf{G}$ suggest the simple explicit forms

$$
W_{1}(\mathbf{F})=w\left(\lambda_{1}, \lambda_{2}, J\right) \quad \text { and } \quad W_{2}(\mathbf{F}, \mathbf{G})=\frac{1}{2} A_{g}\left(\left|\mathbf{g}_{1}\right|^{2}+\left|\mathbf{g}_{2}\right|^{2}\right)+\frac{1}{2} A_{\Gamma}|\boldsymbol{\Gamma}|^{2},
$$

where $A_{g}$ and $A_{\Gamma}$ are positive constants. This is consistent with the model adopted by Spencer and Soldatos [18], who assumed, in the case of a single family of fibers, that the dependence of the strain energy on $\mathbf{G}$ occurs through $\mathbf{g}$. A two-scale model for constructing the strain energy $w$ from microstructural considerations of crimp is given in [1] for the case when $w_{J}$ is negligible.

The energy $W_{1}$ is appropriate for small fiber strains and is easily shown to exhibit orthotropic symmetry [1]. That is, $W_{1}$ remains invariant if $\overline{\mathbf{C}}=\mathbf{H}^{t} \mathbf{C H}$ is substituted in place of $\mathbf{C}$ for $\mathbf{H} \in\left\{ \pm \mathbf{D}_{1} \otimes \mathbf{D}_{1} \pm \mathbf{D}_{2} \otimes \mathbf{D}_{2}\right\}$, with any combination of signs, with the proviso that $J>0$; i.e., that $\lambda_{\alpha}>0$ and $\gamma \in(-\pi / 2, \pi / 2)$. Then, $J$ is determined by the $\lambda_{\alpha}$ and $|\sin \gamma|$, which are orthotropic invariants [1]. Similarly, every term in the function $W_{2}$, which is independent of $\mathbf{F}$ when expressed as a function of $\mathbf{F}$ and $\mathbf{G}$, remains invariant if $G_{i A B}$ is replaced by $\bar{G}_{i A B}=G_{i C D} H_{C A} H_{D B}$. The response of the fabric therefore conforms to orthotropic symmetry relative to the fiber axes in the reference configuration. This is an example of homogeneous symmetry in the general theory of material symmetry for second-grade materials [19] (see also [20]). 
Experiments on fabrics $[21,22]$ are invariably limited to small extensional fiber strains, and indicate that the shear energy is relatively insensitive to the shear angle $\gamma$ at small angles, while increasing sharply as $\gamma \rightarrow \pm \pi / 2$. These limits correspond to fiber collapse, a condition that is not possible in actual fabrics due to the finite widths of the constituent yarns. Bearing in mind applications involving fiber stretches close to unity, we thus assume that $w \rightarrow \infty$ as $J \rightarrow 0$, and that $w_{J}$ is small when $J$ is close to unity.

The function $W_{2}$ is non-negative and vanishes if and only if $\mathbf{G}$ vanishes (cf. (21) and $\left.(34)_{2}\right)$; accordingly, it is a positive-definite, quadratic-and hence convex-function of $\mathbf{G}$. Moreover, the convexity of $W$ with respect to $\mathbf{G}$ is unobjectionable on physical grounds; this stands in contrast to the well-known objections raised against convexity with respect to $\mathbf{F}$ in the conventional first-gradient theory of elasticity. In fact, the direct method of the Calculus of Variations yields the unqualified existence of minimizers in the present theory under a variety of boundary conditions [23].

It is possible to consider a refined model that separates effects such as (tangential) fiber stretch gradient and bending in the strain-energy function. For example, terms like $|\mathbf{g}|^{2}$ in (34) $)_{2}$ may be replaced by $\alpha\left(\lambda^{\prime}\right)^{2}+\beta \kappa^{2}$, with $\alpha$ and $\beta$ positive constants.

(b) A strain-energy function based on the gradient of strain.

There are many alternatives to (34) $)_{2}$ that may be appropriate for fabrics. One that preserves convexity with respect to $\mathbf{G}$ - and hence well-posedness-is given by (34) 1 but with $(34)_{2}$ replaced by

$$
W_{2}(\mathbf{F}, \mathbf{G})=\frac{1}{2} A_{\lambda}\left(\left|\nabla \lambda_{1}\right|^{2}+\left|\nabla \lambda_{2}\right|^{2}\right)+\frac{1}{2} A_{S}|\nabla S|^{2},
$$

where $A_{\lambda}$ and $A_{S}$ are positive constants. This is determined directly by the gradient of $\mathbf{C}$ and thus furnishes an obvious candidate for the gradient energy. Moreover, each of the terms in this expression may easily be shown to remain invariant under the symmetry group for orthotropy (for example, $|\nabla S|$ remains invariant if $\mathbf{C}$ is replaced by $\overline{\mathbf{C}}$ in the definition of $S$ ).

This energy clearly vanishes if $\mathbf{G}$ vanishes. To show that it vanishes only if $\mathbf{G}$ vanishes, we recall that $\lambda_{1,2}$ and $\lambda_{2,1}$ vanish if and only if $\boldsymbol{\Gamma}$ vanishes (provided that $J \neq 0$ ). Then, the gradients $\nabla \lambda_{\alpha}$ vanish if and only if $\boldsymbol{\Gamma}=\mathbf{0}$ with $\mathbf{g}_{1}=\lambda_{1} \kappa_{1} \boldsymbol{v}_{1}$ and $\mathbf{g}_{2}=\lambda_{2} \kappa_{2} \boldsymbol{v}_{2}$. In this case it is straightforward to show, using (31) and (32), that $\nabla S=J\left(\kappa_{1} \mathbf{D}_{1}-\kappa_{2} \mathbf{D}_{2}\right)$, and thus that $W_{2}$ vanishes if and only if $\left\{\mathbf{g}_{1}, \mathbf{g}_{2}, \boldsymbol{\Gamma}\right\}$ vanishes; this is enough to ensure that $W_{2}$ vanishes only if $\mathbf{G}$ vanishes. In the general case, $\nabla \lambda_{\alpha}$ and $\nabla S$ are easily shown to be linear functions of $\mathbf{G}$, so that (35) furnishes a positive-definite, quadratic — and hence convex-function of $\mathbf{G}$.

\subsection{Response Functions}

To compute the response functions $\partial W / \partial F_{i A}$ and $\partial W / \partial G_{i A B}$ for use in the Euler equations and natural boundary conditions (see the Appendix), we use the chain rule in the form

$$
\partial W / \partial F_{i A} \dot{F}_{i A}+\partial W / \partial G_{i A B} \dot{G}_{i A B}=\dot{W}=\dot{W}_{1}+\dot{W}_{2},
$$

where the superposed dots refer to derivatives with respect to a parameter that labels a oneparameter family of deformations. For example,

$$
\dot{W}_{1}=w_{1} \dot{\lambda}_{1}+w_{2} \dot{\lambda}_{2}+w_{J} \dot{J},
$$

where $w_{\alpha}=\partial w / \partial \lambda_{\alpha}$. To proceed we use (28) to obtain

$$
J \dot{J}=\lambda_{2}^{2} \lambda_{1} \dot{\lambda}_{1}+\lambda_{1}^{2} \lambda_{2} \dot{\lambda}_{2}-S \dot{S},
$$


where

$$
\dot{S}=\dot{\mathbf{F}} \mathbf{D}_{1} \cdot \mathbf{F} \mathbf{D}_{2}+\mathbf{F} \mathbf{D}_{1} \cdot \dot{\mathbf{F}} \mathbf{D}_{2}=\left(\mathbf{d}_{1} \otimes \mathbf{D}_{2}+\mathbf{d}_{2} \otimes \mathbf{D}_{1}\right) \cdot \dot{\mathbf{F}}
$$

and

$$
\lambda_{1} \dot{\lambda}_{1}=\mathbf{F D}_{1} \cdot \dot{\mathbf{F}} \mathbf{D}_{1}=\mathbf{d}_{1} \otimes \mathbf{D}_{1} \cdot \dot{\mathbf{F}} .
$$

Using a similar formula for $\lambda_{2} \dot{\lambda}_{2}$, we derive

$$
\begin{aligned}
W_{\mathbf{F}}= & \left(\lambda_{1}^{-1} w_{1}+\lambda_{2}^{2} J^{-1} w_{J}\right) \mathbf{d}_{1} \otimes \mathbf{D}_{1}+\left(\lambda_{2}^{-1} w_{2}+\lambda_{1}^{2} J^{-1} w_{J}\right) \mathbf{d}_{2} \otimes \mathbf{D}_{2} \\
& -w_{J} \tan \gamma\left(\mathbf{d}_{1} \otimes \mathbf{D}_{2}+\mathbf{d}_{2} \otimes \mathbf{D}_{1}\right),
\end{aligned}
$$

where $W_{\mathbf{F}}$ is the tensor with components $\partial W / \partial F_{i A}$.

Proceeding from $(34)_{2}$ in the same way, we have

$$
\dot{W}_{2}=A_{g}\left(\mathbf{g}_{1} \cdot \dot{\mathbf{g}}_{1}+\mathbf{g}_{2} \cdot \dot{\mathbf{g}}_{2}\right)+A_{\Gamma} \boldsymbol{\Gamma} \cdot \dot{\boldsymbol{\Gamma}}
$$

where

$$
\mathbf{g} \cdot \dot{\mathbf{g}}=g_{i} D_{A} D_{B} \dot{G}_{i A B} .
$$

Combining this with

$$
\boldsymbol{\Gamma} \cdot \dot{\boldsymbol{\Gamma}}=\Gamma_{i} D_{A}^{(1)} D_{B}^{(2)} \dot{G}_{i A B}
$$

and the symmetry condition (17), we obtain

$$
W_{\mathbf{G}}=A_{g}\left(\mathbf{g}_{1} \otimes \mathbf{D}_{1} \otimes \mathbf{D}_{1}+\mathbf{g}_{2} \otimes \mathbf{D}_{2} \otimes \mathbf{D}_{2}\right)+\frac{1}{2} A_{\Gamma} \boldsymbol{\Gamma} \otimes\left(\mathbf{D}_{1} \otimes \mathbf{D}_{2}+\mathbf{D}_{2} \otimes \mathbf{D}_{1}\right),
$$

where $W_{\mathbf{G}}$ is the tensor with components $\partial W / \partial G_{i A B}$.

Substitution of these expressions into (72) ${ }_{1-3}$ delivers edge tractions, double forces and corner forces in terms of the deformation of the fabric.

For the alternative energy defined by (35), the response functions are complicated considerably by the fact that $W_{2}$ now depends on $\mathbf{F}$. For example, the stretch of a single yarn family is determined by

$$
\lambda^{2}=F_{i A} F_{i B} D_{A} D_{B} .
$$

Its gradient has components $\lambda_{, C}$, where

$$
\lambda \lambda_{, C}=d_{i} D_{A} G_{i A C}, \quad \text { or } \quad \lambda_{, C}=\tau_{i} D_{A} G_{i A C} .
$$

This has the variational derivative

$$
\dot{\lambda}_{, C}=\tau_{i} D_{A} \dot{G}_{i A C}+\dot{\tau}_{i} D_{A} G_{i A C},
$$

where

$$
\dot{\lambda} \tau_{i}+\lambda \dot{\tau}_{i}=\dot{d}_{i}=\dot{F}_{i B} D_{B}
$$

The latter furnishes

$$
\dot{\tau}_{i}=\lambda^{-1} \Pi_{i j} \dot{F}_{j B} D_{B}, \quad \text { where } \Pi_{i j}=\delta_{i j}-\tau_{i} \tau_{j}
$$


in which $\delta_{i j}$ is the (two-dimensional) Kronecker delta; thus, $\Pi_{i j}=v_{i} v_{j}$. Combining these results, we arrive at the variational derivative

$$
\left(\frac{1}{2}|\nabla \lambda|^{2}\right)=\lambda_{, C} \dot{\lambda}_{, C}=\tau_{i} D_{A} \lambda_{, B} \dot{G}_{i A B}+\lambda^{-1} \Pi_{i j} G_{j B C} D_{B} \lambda_{, C} D_{A} \dot{F}_{i A}
$$

Next, we use $(10)_{2}$ to write the shear strain as

$$
S=F_{i A} F_{i B} D_{A}^{(1)} D_{B}^{(2)}
$$

Its gradient is

$$
S_{, C}=\left[D_{A}^{(1)} D_{B}^{(2)}+D_{A}^{(2)} D_{B}^{(1)}\right] G_{i A C} F_{i B},
$$

with variational derivative

$$
\dot{S}_{, C}=\left[D_{B}^{(1)} D_{A}^{(2)}+D_{B}^{(2)} D_{A}^{(1)}\right] G_{i B C} \dot{F}_{i A}+\left[D_{A}^{(1)} d_{i}^{(2)}+D_{A}^{(2)} d_{i}^{(1)}\right] \dot{G}_{i A C}
$$

Finally,

$$
\begin{aligned}
\left(\frac{1}{2}|\nabla S|^{2}\right)= & S_{, C} \dot{S}_{, C}=\left[D_{A}^{(2)} D_{B}^{(1)}+D_{A}^{(1)} D_{B}^{(2)}\right] G_{i B C} S_{, C} \dot{F}_{i A} \\
& +\left[d_{i}^{(1)} D_{A}^{(2)} S_{, B}+d_{i}^{(2)} D_{A}^{(1)} S_{, B}\right] \dot{G}_{i A B} .
\end{aligned}
$$

We thus obtain the rather unwieldy expressions

$$
\begin{aligned}
\partial W / \partial F_{i A}= & Q_{i A}+A_{\lambda}\left\{\lambda_{1}^{-1} \Pi_{i j}^{(1)} G_{j B C} D_{B}^{(1)} \lambda_{1, C} D_{A}^{(1)}+\lambda_{2}^{-1} \Pi_{i j}^{(2)} G_{j B C} D_{B}^{(2)} \lambda_{2, C} D_{A}^{(2)}\right\} \\
& +A_{S}\left[D_{A}^{(2)} D_{B}^{(1)}+D_{A}^{(1)} D_{B}^{(2)}\right] G_{i B C} S_{, C}
\end{aligned}
$$

and

$$
\begin{aligned}
\partial W / \partial G_{i A B}= & \frac{1}{2} A_{\lambda}\left\{\tau_{i}^{(1)}\left[D_{A}^{(1)} \lambda_{1, B}+\lambda_{1, A} D_{B}^{(1)}\right]+\tau_{i}^{(2)}\left[D_{A}^{(2)} \lambda_{2, B}+\lambda_{2, A} D_{B}^{(2)}\right]\right\} \\
& +\frac{1}{2} A_{S}\left\{d_{i}^{(1)}\left[D_{A}^{(2)} S_{, B}+S_{, A} D_{B}^{(2)}\right]+d_{i}^{(2)}\left[D_{A}^{(1)} S_{, B}+S_{, A} D_{B}^{(1)}\right]\right\},
\end{aligned}
$$

where $Q_{i A}$ are the components of the right-hand side of (41).

The formulation defined by (34) is seen to furnish by far the more tractable alternative. Given the paucity of experimental data on gradient effects in fabrics, there hardly seems to be any reason to consider (35), despite its simple interpretation in terms of the gradient of strain.

Implementation of the proposed model is underway and our intention is to report on some applications of it elsewhere.

Acknowledgements D. Steigmann gratefully acknowledges his appointment as a Visiting Research Professor at the University of Rome 'La Sapienza' during the course of this research. He is also grateful for support provided by the Powley Fund for ballistics research. 


\section{Appendix: Euler Equations and Boundary Conditions}

The derivation of the Euler equations and boundary conditions in second gradient elasticity is well known [24-29]. It is reproduced here for the sake of completeness, and for the purpose of constitutively relating admissible loads to the deformation. To this end, we adopt the framework of the virtual-work statement

$$
\dot{E}=P \text {, }
$$

where the superposed dot refers to the variational—or Gateâux—derivative,

$$
E=\int_{\Omega} W(\mathbf{F}, \mathbf{G}) d a
$$

is the strain energy and $P$ is the virtual power of the edge loads, the form of which is made explicit below. We note that conservative loads are those for which there exists a potential $L$ such that $P=\dot{L}$, and in this case the problem is to minimize the potential energy $E-L$.

We have

$$
\dot{E}=\int_{\Omega} \dot{W}(\mathbf{F}, \mathbf{G}) d a,
$$

where

$$
\dot{W}=\partial W / \partial F_{i A} u_{i, A}+\partial W / \partial G_{i A B} u_{i, A B}
$$

and $\mathbf{u}=\dot{\chi}$ is the variation of the position field. Writing

$$
\begin{aligned}
\partial W / \partial F_{i A} u_{i, A} & =\left(\partial W / F_{i A} u_{i}\right)_{, A}-u_{i}\left(\partial W / \partial F_{i A}\right)_{, A} \text { and } \\
\partial W / \partial G_{i A B} u_{i, A B} & =\left(\partial W / \partial G_{i A B} u_{i, A}\right)_{, B}-u_{i, A}\left(\partial W / \partial G_{i A B}\right)_{, B},
\end{aligned}
$$

we then have

$$
\dot{E}=\int_{\Omega} P_{i A} u_{i, A} d a+\int_{\partial \Omega}\left(\partial W / \partial G_{i A B}\right) u_{i, A} N_{B} d s,
$$

where $\mathbf{N}$ is the rightward unit normal to the boundary curve $\partial \Omega$ in the sense of the GreenStokes theorem, and

$$
P_{i A}=\partial W / \partial F_{i A}-\left(\partial W / \partial G_{i A B}\right)_{, B} .
$$

Decomposing this as in $(62)_{1}$ furnishes

$$
\dot{E}=\int_{\partial \Omega}\left[u_{i} P_{i A} N_{A}+\left(\partial W / \partial G_{i A B}\right) u_{i, A} N_{B}\right] d s-\int_{\Omega} u_{i} P_{i A, A} d a,
$$

and hence the Euler equation

$$
P_{i A, A}=0,
$$

which holds in $\Omega$. With this satisfied we then have

$$
\dot{E}=\int_{\partial \Omega}\left[u_{i} P_{i A} N_{A}+\partial W / \partial G_{i A B}\left(T_{A} N_{B} u_{i}^{\prime}+N_{A} N_{B} u_{i, N}\right)\right] d s,
$$


where use has been made of the normal-tangential decomposition

$$
\nabla \mathbf{u}=\mathbf{u}^{\prime} \otimes \mathbf{T}+\mathbf{u}_{, N} \otimes \mathbf{N}
$$

in which $\mathbf{T}=\mathbf{X}^{\prime}(s)=\mathbf{k} \times \mathbf{N}$ is the unit tangent to $\partial \Omega$; and $\mathbf{u}^{\prime}=d \mathbf{u}(\mathbf{X}(s)) / d s$ and $\mathbf{u}_{, N}$ respectively are the tangential and normal derivatives of $\mathbf{u}$ on $\partial \Omega$. We now substitute

$$
\left(\partial W / \partial G_{i A B}\right) T_{A} N_{B} u_{i}^{\prime}=\left(\partial W / \partial G_{i A B} T_{A} N_{B} u_{i}\right)^{\prime}-\left(\partial W / \partial G_{i A B} T_{A} N_{B}\right)^{\prime} u_{i}
$$

and thereby arrive at

$$
\begin{aligned}
\dot{E}= & \int_{\partial \Omega} u_{i}\left[P_{i A} N_{A}-\left(\partial W / \partial G_{i A B} T_{A} N_{B}\right)^{\prime}\right] d s+\int_{\partial \Omega} u_{i, N}\left(\partial W / \partial G_{i A B} N_{A} N_{B}\right) d s \\
& -\sum u_{i}\left[\partial W / \partial G_{i A B} T_{A} N_{B}\right],
\end{aligned}
$$

where the square bracket refers to the forward jump as a corner of the boundary is traversed. That is, $[\cdot]=(\cdot)_{+}-(\cdot)_{-}$, where the subscripts ${ }_{ \pm}$identify the limits as a corner located at arclength station $s$ is approached through larger and smaller values of arclength, respectively; and the sum refers to the collection of all corners. Here we assume the boundary to be piecewise smooth in the sense that its tangent is piecewise continuous.

We conclude from (58) that admissible powers are of the form

$$
P=\int_{\partial \Omega_{t}} t_{i} u_{i} d s+\int_{\partial \Omega_{m}} m_{i} u_{i, N} d s+\sum_{*} f_{i} u_{i}
$$

where

$$
\begin{aligned}
t_{i} & =P_{i A} N_{A}-\left(\partial W / \partial G_{i A B} T_{A} N_{B}\right)^{\prime}, \quad m_{i}=\partial W / \partial G_{i A B} N_{A} N_{B} \quad \text { and } \\
f_{i} & =-\left[\partial W / \partial G_{i A B} T_{A} N_{B}\right]
\end{aligned}
$$

are the edge traction, edge double force and the corner force, respectively. Here, $\partial \Omega_{t}$ and $\partial \Omega_{m}$ respectively are parts of $\partial \Omega$ where $\chi_{i}$ and $\chi_{i, N}$ are not assigned, and the starred sum refers to corners where position is not assigned. We suppose that $\chi_{i}$ and $\chi_{i, N}$ are assigned on $\partial \Omega \backslash \partial \Omega_{t}$ and $\partial \Omega \backslash \partial \Omega_{m}$, respectively, and that position is assigned at the corners not included in the starred sum.

A simple example of conservative loading is furnished by the potential

$$
L=\int_{\partial \Omega_{t}} t_{i} \chi_{i} d s+\int_{\partial \Omega_{m}} m_{i} \chi_{i, N} d s+\sum_{*} f_{i} \chi_{i}
$$

in which $t_{i}, m_{i}$ and $f_{i}$ are all independent of the deformation.

To interpret the double force in mechanical terms, we consider the special case in which no kinematical data are assigned anywhere on $\partial \Omega$, so that rigid-body deformations are kinematically admissible. The variational derivative of such a deformation is expressible in the form $\mathbf{u}=\omega \times \chi+\mathbf{a}$, where $\mathbf{a}$ and $\boldsymbol{\omega}$ are spatially uniform vectors. Because the strain-energy function is invariant under such deformations, we have $\dot{E}=0$ and (58) reduces to $P=0$; i.e.,

$$
\mathbf{a} \cdot\left(\int_{\partial \Omega} \mathbf{t} d s+\sum \mathbf{f}_{i}\right)+\omega \cdot\left[\int_{\partial \Omega}\left(\chi \times \mathbf{t}+\chi_{, N} \times \mathbf{m}\right) d s+\sum \chi_{i} \times \mathbf{f}_{i}\right]=0,
$$


where $\chi_{i}=\chi\left(\mathbf{X}_{i}\right)$. Because the rigid motion may be arbitrary we then have

$$
\int_{\partial \Omega} \mathbf{t} d s+\sum \mathbf{f}_{i}=\mathbf{0} \quad \text { and } \quad \int_{\partial \Omega}\left(\boldsymbol{\chi} \times \mathbf{t}+\chi_{, N} \times \mathbf{m}\right) d s+\sum \chi_{i} \times \mathbf{f}_{i}=\mathbf{0} .
$$

It follows that $\chi_{, N} \times \mathbf{m}$ is a distribution of edge couples. It is interesting that these couples are configuration dependent in the example of conservative loading described by (73).

\section{References}

1. Nadler, B., Papadopoulos, P., Steigmann, D.J.: Convexity of the strain-energy function in a two-scale model of ideal fabrics. J. Elast. 84, 223-244 (2006)

2. Buckley, C.P., Lloyd, D.W., Konopasek, M.: On the deformation of slender filaments with planar crimp: theory, numerical solution and applications to tendon collagen and textile materials. Proc. R. Soc. Lond. Ser. A, Math. Phys. Sci. 372, 33-64 (1980)

3. Warren, W.E.: The elastic properties of woven polymeric fabric. Polym. Eng. Sci. 30, 1309-1313 (1990)

4. Nadler, B., Papadopoulos, P., Steigmann, D.J.: Multi-scale constitutive modeling and numerical analysis of fabric material. Int. J. Solids Struct. 43, 206-221 (2006). Corrigendum: IJSS 43, 6326-6327 (2006)

5. Ferretti, M., Madeo, A., dell'Isola, F., Boisse, P.: Modeling the onset of shear boundary layers in fibrous composite reinforcements by second-gradient theory. Z. Angew. Math. Phys. (2013). doi:10.1007/s00033-013-0347-8

6. Nadler, B., Steigmann, D.J.: A model for frictional slip in woven fabrics. C. R., Méc. 331, 797-804 (2003)

7. Coleman, B.D.: Necking and drawing in polymeric fibers under tension. Arch. Ration. Mech. Anal. 83, 115-137 (1983)

8. Coleman, B.D., Newman, D.C.: On the rheology of cold drawing: I. Elastic materials. J. Polym. Sci., Part B, Polym. Phys. 26, 1801-1822 (1988)

9. Pipkin, A.C.: Some developments in the theory of inextensible networks. Q. Appl. Math. 38, 343 (1980)

10. Pipkin, A.C.: Plane traction problems for inextensible networks. Q. J. Mech. Appl. Math. 34, 415 (1981)

11. Pipkin, A.C.: Equilibrium of Tchebychev nets. Arch. Ration. Mech. Anal. 85, 81 (1984)

12. Steigmann, D.J., Pipkin, A.C.: Equilibrium of elastic nets. Philos. Trans. R. Soc. Lond. A 335, 419-454 (1991)

13. Kuznetsov, E.N.: Underconstrained Structural Systems. Springer, Berlin (1991)

14. Alibert, J., Seppecher, P., dell'Isola, F.: Truss modular beams with deformation energy depending on higher displacement gradients. Math. Mech. Solids 8, 51-73 (2003)

15. Seppecher, P., Alibert, J.-J., dell' Isola, F.: Linear elastic trusses leading to continua with exotic mechanical interactions. Journal of Physics: Conference Series 319 (2011)

16. Wang, W.-B., Pipkin, A.C.: Inextensible networks with bending stiffness. Q. J. Mech. Appl. Math. 39, 343-359 (1986)

17. Wang, W.-B., Pipkin, A.C.: Plane deformations of nets with bending stiffness. Acta Mech. 65, 263-279 (1986)

18. Spencer, A.J.M., Soldatos, K.P.: Finite deformations of fibre-reinforced elastic solids with fibre bending stiffness. Int. J. Non-Linear Mech. 42, 355-368 (2007)

19. Murdoch, A.I.: Symmetry considerations for materials of second grade. J. Elast. 9, 43-50 (1979)

20. Elzanowski, M., Epstein, M.: The symmetry group of second-grade materials. Int. J. Non-Linear Mech. 27, 635-638 (1992)

21. Cusick, G.E.: The response of fabric to shearing forces. J. Text. Inst. 52, T395-T406 (1961)

22. Cao, J., Akkerman, R., Boisse, P., Chen, J., Cheng, H.S., De Graaf, E.F., Zhu, B.: Characterization of mechanical behavior of woven fabrics: experimental methods and benchmark results. Composites, Part A, Appl. Sci. Manuf. 39, 1037-1053 (2008)

23. Ball, J.M., Currie, J.C., Olver, P.J.: Null Lagrangians, weak continuity, and variational problems of arbitrary order. J. Funct. Anal. 41, 135-174 (1981)

24. Toupin, R.A.: Theories of elasticity with couple stress. Arch. Ration. Mech. Anal. 17, 85-112 (1964)

25. Mindlin, R.D., Tiersten, H.F.: Effects of couple-stresses in linear elasticity. Arch. Ration. Mech. Anal. 11, 415-448 (1962)

26. Koiter, W.T.: Couple-stresses in the theory of elasticity. Proc. K. Ned. Akad. Wet. 67, 17-44 (1964)

27. Germain, P.: The method of virtual power in continuum mechanics, part 2: microstructure. SIAM J. Appl. Math. 25, 556-575 (1973) 
28. dell'Isola, F., Seppecher, P., Madeo, A.: Beyond Euler-Cauchy continua: the structure of contact actions in N-th gradient generalized continua: a generalization of the Cauchy tetrahedron argument. In: Variational Models and Methods in Solid and Fluid Mechanics. CISM Courses and Lectures, vol. 535, pp. 17-106 (2012)

29. dell'Isola, F., Seppecher, P., Madeo, A.: How contact interactions may depend on the shape of Cauchy cuts in N-th gradient continua: approach “à la D'Alembert”. Z. Angew. Math. Phys. 63, 1119-1141 (2012) 